\title{
Balanceakt zwischen Mißtrauen und Kooperation
}

\author{
Im internationalen Wettbewerb der Standorte spielen auch Umweltschutzbe- \\ stimmungen eine bedeutende Rolle. Um globale Umweltprobleme gemeinsam \\ lösen zu können, herrscht besonderer Regelungsbedarf, Welche Vorschläge hat \\ der Ausschuß zu Handel und Umwelt der WTO vorgelegt? Wie sind diese zu \\ beurteilen?
}

$\mathrm{D}$

Von Carsten Helm ie Globalisierung der Wirtschaft läßt auch den Faktor Umweltschutz zunehmend als wichtigen Bestandteil im ,Wettbewerb der Standorte“ erscheinen. Häufig wird hier einseitig die Kostenseite betont, während die positiven Effekte strenger Umweltschutzbestimmungen wie ,firstmover-Vorteile“ und „Umweltqualität als Standortfaktor" weniger Beachtung finden. Aber ebenso läßt sich die Befürchtung, daß die Globalisierung zu einer Anpassung von Umweltstandards nach unten fuihrt, bisher nicht belegen (1).Internationale Umweltprobleme weisen jedoch bestimmte Eigenschaften auf, die einen besonderen Regelungsbedarf mit sich bringen. Im Mittelpunkt des Interesses steht die Reform der 1994 gegründeten Welthandelsorganisation (WTO), nicht zuletzt da sie aufgrund ihres ausgebildeten Streitschlichtungsverfahrens und dem Machtpotential von Handelssanktionen als einer der wenigen ,rauchenden Colts" der internationalen Beziehungen gilt (2).

\section{Rechtfertigung von Handels- beschränkungen}

Bei nationalen Umweltproblemen kann die Externalisierung der bei der Produktion und beim Konsum von Gütern entstehenden Umweltschäden durch staatliche umweltpolitische Maßnahmen unterbunden werden, etwa durch Ökosteuern, Umweltzertifikate oder ordnungsrechtliche Vorschriften. Internationaler Handel mag zwar die bestehenden Defizite verstärken, er ist jedoch nicht ursächlich für sie verantwortlich.

Bei internationalen Umweltproblemen stoßen nationale Maßnahmen hingegen an Grenzen, da zumindest ein Teil der Umweltschäden durch Aktivitäten in anderen Ländern verursacht wird (3). Schlimmer noch, die Wirksamkeit nationaler umweltpolitischer Maßnahmen kann durch internationale Handelseffekte teilweise aufgehoben werden, beispielsweise durch die Verlagerung verschmutzungsintensiver Produktion in Länder ohne entsprechende Regelungen. In diesem Fall kann ein „ökozoll“ - eine ,grenzüberschreitende Verschmutzungsabgabe“ (4) gerechtfertigt sein, da er die globalen Wirtschaftsbeziehungen nicht zusätzlich verzerrt, sondern die Verzerrung durch in den Preisen nicht berücksichtigte grenzüberschreitende Verschmutzung ausgleicht.

Eine solche einseitige Maßnahme beeinflußt jedoch nur die grenzïberschreitenden Emissionen der Produktion im Ausland für den Export und darin liegt ihre Schwäche. Insgesamt ließe sich also durch internationale Kooperation mehr globaler Umweltschutz erreichen. Das heißt, daß transnationale Verschmutzungsabgaben immer nur eine zweitbeste Politikmaßnahme darstellen und nur dort zu empfehlen sind, wo kooperative Lösungen scheitern.

Selbst wenn alle Staaten von einem internationalen Umweltabkommen profitierten, wäre dessen Zustandekommen beziehungsweise seine Stabilität durch Trittbrettfahrer bedroht: Angenommen aus einer Koalition miteinander kooperierender Staaten würde einer austreten, um von einer Trittbrettfahrerposition zu profitieren. Ein Abbruch der Kooperation durch die übrigen Staaten wäre zwar eine wirkungsvolle Abschreckung, aber eine solche Drohung ist nicht glaubwürdig, da sich die verbliebenen Staaten hierdurch selbst schaden würden. Gerade bei drängenden Umweltproblemen mit hohen Kooperationsgewinnen wäre das Drohpotential gering, da in diesem Fall der Anreiz für die verbleibenden Koalitionäre groß ist, weiterhin substantielle kooperative Reduktionsmaßnahmen durchzuführen. Handelsbeschränkungen könnten hier das notwendige

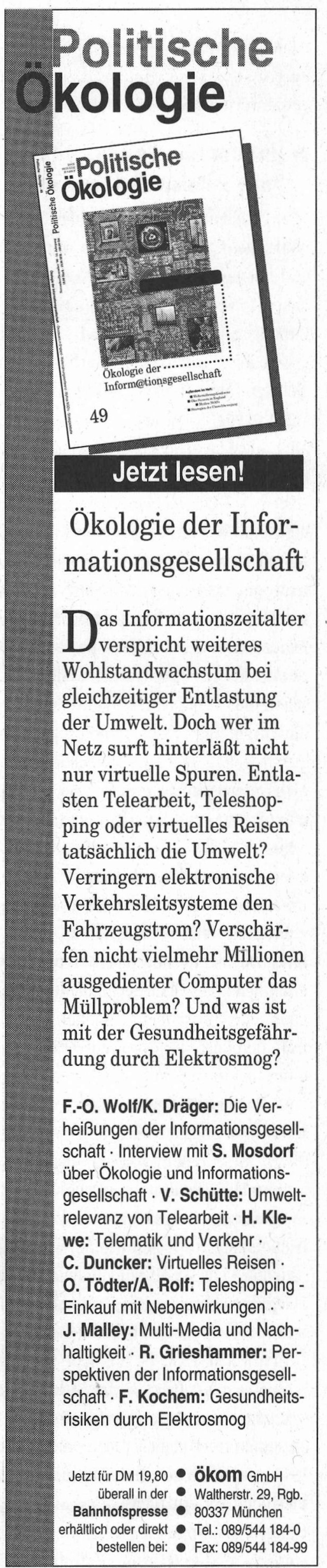


Sanktionspotential liefern, um die Stabilität pareto-superiorer Kooperationslösungen $\mathrm{zu}$ gewährleisten (5).

\section{- Internationale Umweltabkom- men - Beispiel Klimaschutz}

Wie verhalten sich die beiden angefiihrten (ökonomischen) Rechtfertigungen von Handelsbeschränkungen bei internationalen Umweltproblemen - Verbesserung der Wirksamkeit unilateraler Umweltschutzmaßnahmen und Sanktionspotential gegenüber Trittbrettfahrerverhalten - zur Praxis globaler Umweltpolitik? Sicherlich gibt es eine Vielzahl von Erklärungen für die Erfölge und Mißerfolge beim Schutz der Ozonschicht und des Klimas. Insbesondere hat sich gezeigt, daß die Kosten des Klimaschutzes ungleich höher sind, während sein Nutzen hinter dem Schleier der wissenschaftlichen Unsicherheit noch recht verschwommen erscheint. Allerdings spielt auch die unterschiedliche Behandlung des internationalen Handles eine wesentliche Rolle.

Der Artikel vier des Montrealer Protokolls zum Schutz der Ozonschicht enthält eine Reihe von Einschränkungen des Handels mit Nicht-Vertragsparteien, die zu verschiedenen Zeitpunkten wirksam wurden (6):

- ein Export- und Importverbot für die kontrollierten Stoffe,

- ein Importverbot für Waren aus Nicht-Vertragsparteien, die die kontrollierten Stoffe enthalten (z.B. Kühlschränke),

- ein mögliches Importverbot für Waren, die mit den kontrollierten Stoffen hergestellt wurden, diese aber nicht enthalten (z.B. mit FCKWs gereinigte elektronische Komponenten),

- der Export von Technologien zur Verwendung oder Herstellung der kontrollierten Stoffe sollte ,entmutigt" werden.

Durch diese Bestimmungen wurde Verlagerungen von FCKW-Industrien in Nicht-Vertragsparteien erfolgreich vorgebeugt und die Anreize zum Trittbrettfahrerverhalten wurden vermindert. Die Bedeutung dieser Bestimmungen betonte der Delegationsleiter der USA, Richard Benedick, als „critical, since they constituted the only enforcement mechanism in the protocol" (7).

In den weit weniger entwickelten Vereinbarungen zum Schutz des Klimas fehlen ähnliche Bestimmungen bisher. Dies beeinträchtigt die Aussichten umfassender Schutzmaßnahmen entscheidend. So erwarten die meisten Studien bei unilateralen Maßnahmen eine Zunahme des Kohlendioxid-(CO2)-Ausstoßes im Ausland, auch wenn die Schätzungen dieses ,,carbon leakage“ je nach Modellannahmen oft weit auseinander liegen: zwischen einigen wenigen Prozent bis zu mehr als 100 Prozent für Industrien mit zunehmenden Skalenerträgen (8). Die sich hieraus ergebenden Probleme lassen sich exemplarisch an der inzwischen gescheiterten europäischen C02/Energieabgabe zeigen. Diese enthielt nämlich zwei Konditionalitätsklauseln, die Ausnahmen für energieintensive Sektoren vorsahen und die Einfïhrung der Abgabe von entsprechenden Maßnahmen in den anderen OECD-Ländern abhängig machten. Hier läßt sich die Angst vor Trittbrettfahrern deutlich ablesen.

Eine Lösung dieses Konflikts ist allerdings wesentlich schwerer als in Fragen des Ozonschutzes. Schließlich geht es hierbei nicht um den Handel mit CO2, sondern um die CO2-Intensität von Prozeß- und Produktionsmethoden. Vor diesen war man jedoch auch beim Schutz der Ozonschicht zurückgeschreckt. So wurde das oben angefiuhrte Importverbot für Waren, die mit den kontrollierten Stoffen hergestellt wurden, diese aber nicht enthalten, von den Vertragsstaaten im November 1993 als zum derzeitigen Zeitpunkt nicht durchfiihrbar eingestuft.

\section{- Vorschläge des Ausschusses zu Handel und Umwelt der WTO}

Was also ist derzeit durchfuihrbar? Die Vereinbarkeit von Handelsbeschränkungen in internationalen Umweltabkommen mit dem multilateralen Handelssystem bildete neben ökolabels den wesentlichen Arbeitsschwerpunkt des 1994 zusammen mit der WTO ins Leben gerufenen Ausschusses zu Handel und Umwelt. Dort wurde bislang ein weites Spektrum unterschiedlicher Vorschläge diskutiert, von denen im folgenden einzelne aufgelistet werden:

- Es bestehe kein Reformbedarf, insbesondere aufgrund der in Artikel XX des GATT (General Agreement on Tariffs and Trade) vorgesehenen Möglichkeit ansonsten GATT-widriger Handelsbeschränkungen zur Erreichung eines übergeordneten öffentlichen Politikziels wie dem Schutz des Lebens und der Gesundheit von Menschen, Tieren und Pflanzen.

- Mit dem eigentlich nur für außergewöhnliche und vorübergehende Umstände vorgesehenen sogenannten Waiver-Ansatz (Artikel IX der WTO) könnten ausgewählte internationale Umweltabkommen ausdrïcklich dem GATT vorangestellt werden.
- Der Schutz der Umwelt könnte den Ausnahmen in Artikel XX(b) ausdrücklich hinzugefiugt werden, möglicherweise in Verbindung mit einer Abschwächung des dort enthaltenen ,Notwendigkeitskriteriums" und statt dessen einer stärkeren Betonung der Zielbestimmung in Artikel XX.

- Durch eine Sonderklausel könnten WTO-Streitschlichtungsausschüsse beauftragt werden, nicht die umweltpolitische Legitimität oder Notwendigkeit von Handelsbeschränkungen in internationalen Umweltabkommen zu untersuchen, sondern nur, ob diese entgegen der Zielbestimmung in Artikel XX diskriminierend oder mit dem Ziel von Handelsvorteilen angewendet werden.

- Es könnten Richtlinien mit allgemeinen Kriterien für die Formulierung von Handelsbeschränkungen in internationalen Umweltabkommen aufgestellt werden, z.B. deren Notwendigkeit, Effektivität oder Proportionalität. In seinem Bericht zur ersten WTO-Ministerkonferenz, die in Singapur vom 9. bis 13. Dezember 1996 stattfand, kommt der Ausschuß zu Handel und Umwelt über eine Aufzählung dieser einzelnen Vorschläge nicht hinaus, wofür er seitens vieler Umweltschutzgruppen und auch einiger Regierungen heftige Kritik erntete. Zudem muß man kritisieren, daß die derzeitige Konzentration auf die Frage, ob in Konfliktfällen die WTO oder internationale Umweltabkommen Vorrang haben, viel zu kurz greift - zumal es oft dieselben Staaten sind, die in beiden Institutionen die Entscheidungen prägen. Vielmehr müßte grundsätzlich gefragt werden, wie sich die gleichermaßen legitimen Ziele einer globalen Umweltpolitik und eines liberalen Handelsregimes in Einklang bringen lassen. Die noch ausstehende Lösung müßte dann sowohl in der WTO als auch in den internationalen Umweltabkommen verankert werden. Auf die Klimapolitik zugespitzt könnte es sich als entscheidende Frage herauskristallisieren, ob und in welchem Umfang Handelsbeschränkungen auf der Grundlage unterschiedlicher Prozeßund Produktionsmethoden erlaubt sein sollen, um Trittbrettfahrer zum Kauf einer Fahrkarte zu bewegen und die Wirksamkeit unilateraler Maßnahmen zu erhöhen. Hier hat das GATT/WTORegime selbst mit widersprüchlichen Regelungen zur Verwirrung beigetragen (2): Zunächst gibt es da einen Grundsatzbericht von 1970, der die Klärung dieses umstrittenen Punkts mit dem Hinweis auf seine damalige Bedeutungslosigkeit vertagte; des weiteren exisitiert eine Ausnahmeklausel, daß Exporte möglicherweise von einer Energiesteuer befreit werden können, deren ver- 


Der Bericht des WTO-Ausschusses zu
Handel und Umwelt
Der Bericht des Ausschusses zu Handel und Umwelt wur
de bereits am 9. November 1996 einstimmig angenom-
men. Handel und Umwelt war daher kein Verhandlungs-
gegenstand auf der vom 9. bis 13. Dezember in
Singapur stattfindenden ersten WTO-Ministerkonferenz.
Der Bericht geht über eine Wiedergabe der von den ein-
zelnen Vertragsstaaten geäuBerten Standpunkte kaum
hinaus und wurde daher besonders von Umweltgruppen,
aber auch einzelnen Regierungen mit Enttäuschung auf-
genommen. Lediglich drei substantielle Empfehlungen las-
sen sich finden:
1. Die WTO sollte den Sekretariaten von internationalen
Umweltabkommen Beobachterstatus im Ausschuß zu
Handel und Umwelt gewähren.
2. Eine Datenbank für handelsbezogene Umweltschutz-
maßnahmen sollte angelegt werden.
3. Der Ausschuß sollte seine Arbeit als permanente Ein-
richtung der WTO fortsetzen.

steckter Platz in der Fußnote eines Anhangs zu einem Nebenabkommen des GATT zur potentiellen Bedeutung dieser Bestimmung aber in keinem Verhältnis steht; und schließlich existieren noch die zwei Streitschlichtungsberichte zum Thunfisch-Delphin-Fall, die von den Vertragsstaaten aber (noch) nicht angenommen wurden.

\section{Gemeinsame aber differenzierte Verpflichtungen}

Soll es $z u$ einer befriedigenden Regelung von Handelsbeschränkungen bei internationalen Umweltproblemen kommen, so müßten zunächst folgende Einsichten allgemein anerkannt werden:

- Erstens: Ziel des GATT/WTO-Regimes kann nicht „Freihandel“ sein, sondern ein Handel frei von wettbewerbsverzerrenden Effekten, der auf der Grundlage komparativer Kostenvorteile stattfindet. Maßnahmen zur Internalisierung externer Effekte - und hierzu können auch grenzüberschreitende Verschmutzungsabgaben zählen - befinden sich aus dieser Perspektive durchaus in Einklang mit dem Ziel eines liberalen Handelssystems.

- Zweitens: Eine nationale Umweltpolitik ist bei internationalen Umweltproblemen nicht möglich, und somit auch der Grundsatz der nationalen Souveränität, die eigene Umweltpolitik zu bestimmen, eine Illusion und als grundlegendes Prinzip ungeeignet. Die entscheidende Frage muß daher lauten, unter welchen Umständen es erlaubt sein sollte, auf die Umweltpolitik anderer Länder Einfluß zu nehmen. Die Einschränkung der nationalen Souveränität in der
Erklärung von Rio durch die Bedingung, nicht die Umwelt anderer Staaten zu schädigen, kann hier als erster Ausgangspunkt dienen. Besonders bei globalen Umweltproblemen, zu denen mehr oder weniger alle Staaten beitragen, liefert es jedoch nur wenig Orientierung.

- Schließlich muß sich jede Regelung den Problemen des protektionistischen Mißbrauchs und einer möglicherweise ungerechten Verteilung der Kosten internationaler Umweltprobleme stellen. Schließlich wäre es unfair, den bisher für das Klimaproblem nicht verantwortlichen Entwicklungsländern über Handelsbeschränkungen einen Teil der Reparaturkosten ohne entsprechende Kompensation zu übertragen, selbst wenn hierdurch die Wirksamkeit der internationalen Klimapolitik gesteigert würde.

Hieraus ergeben sich für die Regelung von Handelsbeschränkungen bei internationalen Umweltfragen drei zentrale Fragen, auf die sich die Diskussion verstärkt konzentrieren sollte:

- Wie streng sollten die Maßstäbe gegen einen protektionistischen Mißbrauch von Handelsbeschränkungen bei internationalen Umweltproblemen sein?

Hier könnten relativ strikte Kriterien wie die Notwendigkeit, Effektivität oder Proportionalität von Maßnahmen angewendet werden; oder die Streitschlichtungsausschüsse könnten ihre Überprïfung auf die Einhaltung der Zielbestimmungen in den Artikel III und XX - keine ungerechtfertigte Diskriminierung und kein versteckter Schutz der inländischen Industrie beschränken. Es ist durchaus denkbar, daß an Handelsbeschränkungen mit einem geringeren internationalen Rückhalt strengere Maßstäbe angelegt werden als an solche im Rahmen quasi universeller Umweltabkommen.

- Was ist eine faire Verteilung der gemeinsamen aber differenzierten Verpflichtungen bei internationalen Umweltproblemen?

Diese Frage geht weit über die Beurteilung handelsbeschränkender Maßnahmen hinaus und wäre in erster Linie im Rahmen internationaler Umweltabkommen zu klären; bei bisher nicht verregelten Umweltproblemen könnten die Praxis der Staatengemeinschaft in ähnlichen Fällen - insbesondere das Völkergewohnheitsrecht oder allgemeine Prinzipien, wie sie in der RioErklärung festgelegt wurden, vorïbergehend als Orientierung dienen. Von besonderer Bedeutung ist hier das Prinzip der gemeinsamen aber differenzierten Verpflichtungen (Prinzip 7 der
Rio-Erklärung), die sich aus der unterschiedlichen Verantwortlichkeit und Fähigkeit der einzelnen Staaten ergeben (6).

- Wer soll in Streitfällen die Definitionsmacht haben?

Eine stärkere Beteiligung von Umweltexperten am WTO-Streitschlichtungsverfahren kann hier nur ein erster Schritt sein. Probleme ergeben sich besonders bei Konflikten zwischen Vertragsparteien und Nicht-Vertragsparteien internationaler Umweltabkommen. Hier könnte die Forderung des Forums Umwelt und Entwicklung deutscher Nichtregierungsorganisationen nach einem übergeordneten, unabhängigen Sreitschlichtungsmechanismus aufgegriffen werden, der neben WTO und UNCTAD auch mit der Umweltpolitik betraute Institutionen wie etwa UNEP (United Nations Environment Programme) und die CSD (Commission for Sustainable Development) mit einbezieht.

\section{Literafur}

1) David Vogel: Trading Up: Consumer and Environmental Regulation in a Global Economy. Harvard University Press, Cambridge Ma 1995

2) Carsten Helm: Sind Freihandel und Umweltschutz vereinbar? Ökologischer Reformbedarf des GATT/WTO-Regimes. edition sigma. Berlin 1995

3) Carsten Helm: Transboundary Environmental Problems and New Trade Rules. in: International Journal of Social Economics, 1996 Vol. 23, №. 8, 29-45.

4) William J. Baumol, Wallace E. Oates: The Theory of Environmental Policy. Prentice-Hall, Eaglewood Cliffs 1975 5) Carsten Helm: Weltumweltpolitik und ökonomische Theorie. in: Udo E. Simonis (Hrsg.): Weltumweltpolitik Grundriß und Bausteine eines neuen Politikfeldes, edition sigma, Berlin 1996, S. 266-287.

- Raimund Krumm: Internationale Umweltpolitik: Eine Analyse aus umweltökonomischer Sicht. Springer, Berlin 1995 6) Frank Biermann: Saving the Atmosphere - International Law, Developing Countries and Air Pollution. Peter Lang, Frankfurt a.M. 1995

7) Richard E. Benedick: Ozone Diplomacy: New Directions in Safeguarding the Planet, Harvard University Press, Cambridge Ma 1991

8) Scott Barrett: Climate Change Policy and International Trade. CSERGE Working Paper GEC 94-12, 1994

\section{Der Autor}

Carsten Helm, MA (Econ), Dipl..Pol., ist wissenschaftlicher Mitarbeiter am Potsdam-Institur für Klimafolgenforschung $e . V$.

Kontakt: Potsdam-Institut für Klimafolgenforschung, Postfach 601203, 14412 Poisdam, Tel. (0331) 288-2557, Fax - 2600 
(c) 20I0 Authors; licensee IÖW and oekom verlag. This is an article distributed under the terms of the Creative Commons Attribution Non-Commercial No Derivates License (http://creativecommons.org/licenses/by-nc-nd/3.o/), which permits unrestricted use, distribution, and reproduction in any medium, provided the original work is properly cited. 